\title{
WILMS' TUMOR IN ADULTS
}

\author{
JOSE M. ALAPONT, JOSE L. PONTONES, JUAN F. JIMENEZ-CRUZ \\ Department of Urology, La Fe University Hospital, Valencia, Spain
}

\begin{abstract}
Wilms' tumor is an uncommon neoplasm in adults. We report the clinical manifestations, complementary explorations, treatment, and results from 3 males aged 16, 21, and 22 years. Computed tomography commonly suggests the diagnosis. Despite its aggressive treatment, such as radical surgery, chemo- and radiotherapy, the prognosis is worse than in children.
\end{abstract}

Key words: kidney; kidney neoplasms; nephroblastoma; adult Int Braz J Urol. 2003; 29: 40-2

\section{INTRODUCTION}

Wilms' tumor is the most common abdominal tumor in children, though in adults it is extremely rare, representing only $0.5 \%$ of all renal neoplasms. To date, 240 cases in adults have been reported in the literature (1).

\section{CASES REPORT}

\section{Case 1}

A 16-year-old male comes to the clinic for asymptomatic hematuria. Ultrasound and CT showed a heterogeneous mass in the left kidney. The fineneedle aspiration biopsy (FNAB) was suggestive of carcinoma or renal hamartoma. A laparotomy was performed and the intraoperative biopsy suggested nephroblastoma (Wilms' tumor). A radical nephrectomy was thus performed. The histopathological report confirmed the diagnosis of Wilms' tumor. The workup for metastasis was negative. Treatment was started with irradiation of the tumor bed (16 Gy) and chemotherapy according to the Society International of Pediatric Oncology (SIOP) 9 protocol. Twelve months later, local and systemic recurrence (lung metastases) were detected. Three new chemotherapy regimens were administered: one with etoposide, carboplatin, ifosfamide, vincristine, and actinomycin$\mathrm{D}$, other with cisplatin and epirubicin, and the third with cisplatin and etoposide. The disease continued to progress and the patient died 36 months after nephrectomy.

\section{Case 2}

A 21-year-old male came to the clinic with pain in the region of the renal fossa and cachexia. CT and angio-MRI (Figure-1A) showed a mass in the right kidney and nodular images in both lung fields. FNAB of the renal mass was suggestive of Wilms' tumor. After a radical nephrectomy with removal of the retroperitoneal mass (Figure-1B), the histopathological study confirmed the diagnosis. Adjuvant chemotherapy was administered according to the SIOP 93-01 protocol, together with radiotherapy (tumor bed, lung fields, and adenopathies). The disease progressed and, after 2 cycles of topotecan, cyclophosphamide and, ifosfamide, the patient died 14 months after nephrectomy. 


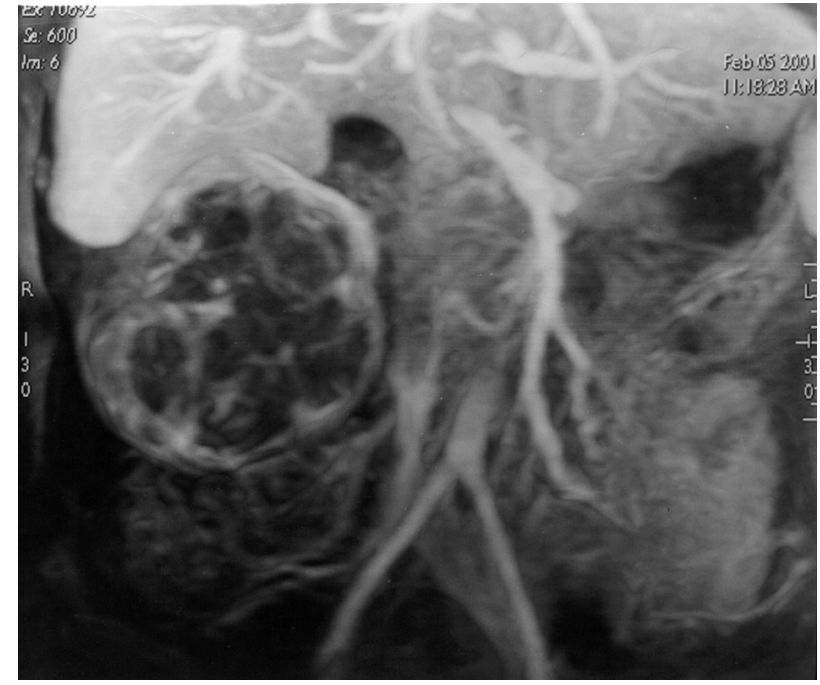

Figure 1 - Angio-MRI demonstrating renal mass and thrombosis of the right renal vein.

\section{Case 3}

A 22-year-old male consulted for hematuria, pain in renal fossa and fever. Ultrasound and CT showed a heterogeneous mass in the right kidney. A radical nephrectomy was performed and the histopathological analysis demonstrated nephroblastoma. Adjuvant radiotherapy (30 Gy) and chemotherapy (6 cycles of vincristine, cisplatin, and doxorubicin) were administered. After a follow-up of 180 months the patient is clinically free of disease.

\section{DISCUSSION}

The diagnostic criteria defining adult nephroblastoma were described by Kilton et al. (2). This disease is difficult to differentiate from renal cell carcinoma based only on imaging techniques, though preoperative diagnosis may be suggestive in about $75-80 \%$ of cases. Ultrasound observation of a rapidly growing abdominal mass in a young patient, with heterogeneous contrast uptake, and surrounded by a pseudocapsule on CT is suggestive of Wilms' tumor. Arteriography characteristically shows a hypovascular mass with neoformed blood vessels exhibiting a zigzag pattern. The histopathological study confirms the diagnosis. The treatment is not well established for adults. Aggressive treatment, in-

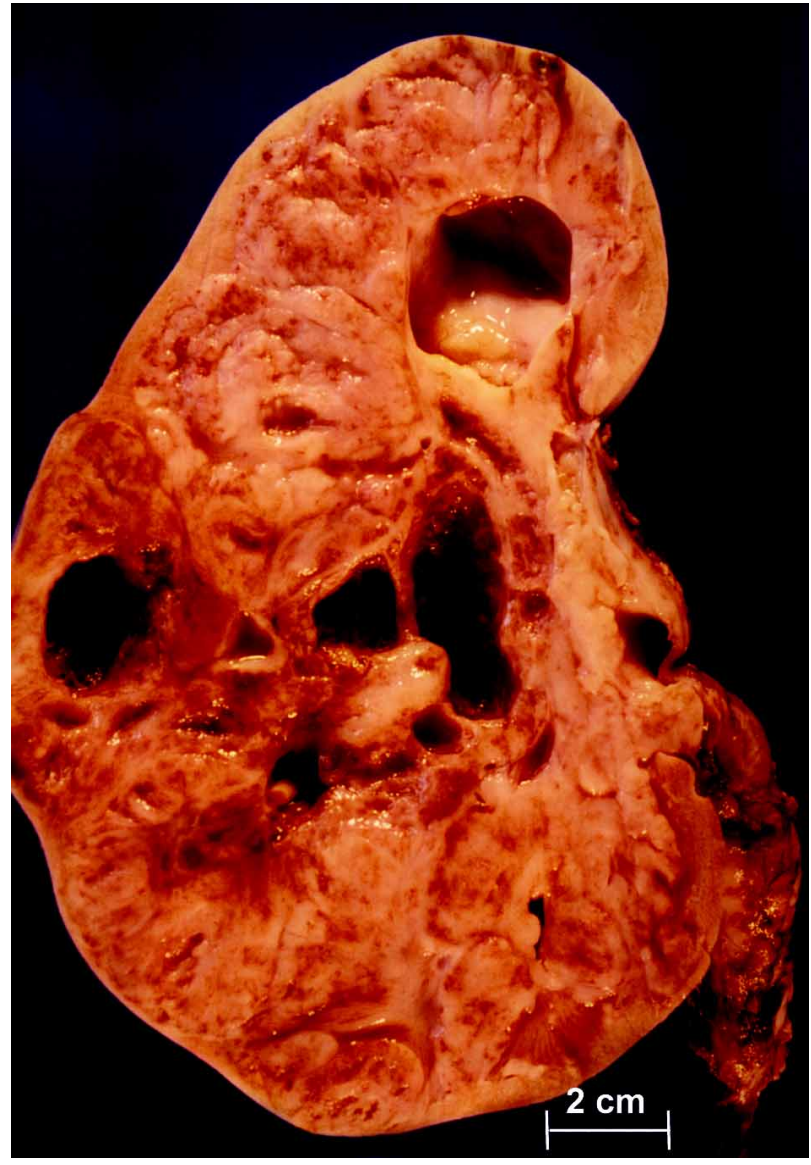

Figure 2 - Macroscopic view of the specimen.

cluding radical surgery, chemotherapy, and irradiation of the tumor bed, is considered necessary. The habitually used chemotherapeutic agents are vincristine, actinomycin-D, doxorubicin and ifosfamide. Satisfactory results have also been published with cisplatin and etoposide in patients with stage IV disease and patients in progression after conventional chemotherapy (3). The prognosis in adults is worse than in children. Our 3 patients were treated with multimodal treatment, and only 1 is alive and free of disease.

\section{REFERENCES}

1. Hentrich MU, Meister P, Brack NG, Lutz LL, Hartenstein RC: Adult Wilms' tumor. Cancer 1995; 75:545-51. 


\section{WILMS' TUMOR IN ADULTS}

2. Kilton L, Matthews MJ, Cohen MH: Adult Wilms' tumor: report of prolonged survival and review of literature. J Urol. 1980; 124:1-5.

\section{Correspondence address:}

Dr. José Miguel Alapon

Service of Urology, La Fe University Hospital

Avda. Campanar 21, 46009

Valencia, Spain

Fax: + 96 386-2600

E-mail:jmalapont@mundofree.com
3. Sparano JA, Beckwith JB, Mitsudo S, Wiernick PH: Complete remission in refractory anaplastic adult Wilms' tumor treated with cisplatin and etoposide. Cancer 1991; 67:956-9.

Received: November 11, 2002 Accepted after revision: January 24, 2003 